\title{
Intuitionistic Fuzzy Hamiltonian Cycle by Index Matrices
}

\author{
Velichka Traneva \\ "Prof. Asen Zlatarov" University \\ "Prof. Yakimov" Blvd, Burgas 8000, Bulgaria \\ Email: veleka13@gmail.com
}

\author{
Stoyan Tranev \\ "Prof. Asen Zlatarov" University \\ "Prof. Yakimov" Blvd, Burgas 8000, Bulgaria \\ Email: tranev@abv.bg
}

\begin{abstract}
In this paper, the algorithm for finding a Hamiltonian cycle in an intuitionistic fuzzy graph (IFG) is proposed, based on the theories of intuitionistic fuzzy sets (IFSs) and of index matrices (IMs). The aim of the paper is to extend the algorithm to find a fuzzy Hamiltonian cycle (FHC) in an IFG to the intuitionistic fuzzy (IFHC) using the IFSs and IMs concepts. An intuitionistic fuzzy graph example about network of Wizz air airlines is modeled by the extended IM to illustrate the proposed algorithm. In the paper also are introduced for the first time three index-type operations over IMs.
\end{abstract}

\section{INTRODUCTION}

A HAMILTONIAN cycle is a cycle through a graph that visits each node exactly once (see [21]). Determining if a graph is Hamiltonian is well known to be NP-complete [20]. Dirac (1952, [6]) described some relations between the degree of the nodes in a graph and the lengths of the circuits contained in it. Ore, Chvatal and Fan have provided the sufficient conditions for a graph to be Hamiltonian (see [7], [19], [23]). Zhao, in 2007, gave better conditions for the existence of Hamiltonian paths in a graph (see [17]).

Nowadays, some parameters of the graph problem may be uncertain due to uncontrollable factors. The fuzzy sets (FSs) of Zadeh appeared in 1963 [18] to deal with this environment. The first idea of a fuzzy graph was described by Kaufman [2]. Rosenfeld [3] developed the theory of fuzzy graphs in 1975. Mordeson and Nair have also proposed another concepts in fuzzy graphs [8]. An algorithm for fuzzy Hamiltonian cycle in a network using adjacency matrix was proposed by Gani and Latha [1]. In 1983, Atanassov proposed the IFSs ([9], [11]), which is an extension of the FSs. The major advantage of IFS over FS is that IFS separates the degree of membership and non-membership of an element.

In this paper, it is proposed for the first time two algorithms for finding Hamiltonian cycle in an intuitionistic fuzzy graph (IFG), based on the concepts of IFSs and of IMs (see [10], [12]). The first algorithm is illustrated with an IFG example about a network of Wiz Air [28].

The rest of this paper is structured as follows: Section 2 describes the related concepts of the IMs, IFSs and IFGs. In the section 2 also are introduced for the first time three index-type

The work is supported by the Ministry of Education and Science under the Programme "Young scientists and postdoctoral students", approved by DCM \# 577/17.08.2018. operations over IMs. In Section 3, we propose an algorithm for determining a Hamiltonian cycle in an IFG, based on the fuzzy algorithm [1], by using the concepts of IMs and IFSs. The effectiveness of the proposed method is demonstrated by an example in Section 4. Section 5 outlines the conclusion and some directions for future research.

\section{BASIC DEFINITIONS OF IMS, INTUITIONISTIC FUZZY LOGIC AND IFG}

This section presents some definitions on intuitionistic fuzzy pairs (IFPs) from (see [5], [11], [15], [25]), on IMs concept from (see [12], [27]) and on IFG (see [4], [12]).

\section{A. Remarks on Intuitionistic Fuzzy (IF) Logic}

The IFP is an object in the form of an ordered pair $\langle a, b\rangle=$ $\langle\mu(p), v(p)\rangle$, where $a, b \in[0,1]$ and $a+b \leq 1$, that is used as an evaluation of a proposition $p$ (see [15]). $\mu(p)$ and $v(p)$ respectively determine the "truth degree" (degree of membership) and "falsity degree" (degree of non-membership). With two IFPs $x=\langle a, b\rangle$ and $y=\langle c, d\rangle$ were defined some basic operations and relations with IFPs

$$
\begin{gathered}
x \wedge_{1} y=\langle\min (a, c), \max (b, d)\rangle ; \\
\left.x \vee_{1} y=\langle\max (a, c)), \min (b, d)\right\rangle ; \\
x \wedge_{2} y=x+y=\langle a+c-a . c, b . d\rangle ; \\
x \vee_{2} y=x \cdot y=\langle a . c, b+d-b . d\rangle ; \\
\neg x=\langle b, a\rangle ; \alpha \cdot x=\left\langle 1-(1-a)^{\alpha}, b^{\alpha}\right\rangle(\alpha \in R) ; \\
x-y=\langle\max (0, a-c), \min (1, b+d, 1-a+c)\rangle
\end{gathered}
$$

and relations with IFPs

$$
\begin{array}{cc}
x \geq y \text { iff } a \geq c \text { and } b \leq d ; & x \leq y \text { iff } a \leq c \text { and } b \geq d ; \\
x \geq \square y \text { iff } a \geq c ; & x \leq \square y \text { iff } a \leq c ; \\
x \geq_{\diamond} y \text { iff } b \leq d ; & x \leq \diamond y \text { iff } b \geq d ; \\
x=y & \text { iff } a=c \text { and } b=d ; \\
x \geq_{R} y & \text { iff } R_{\langle a, b\rangle} \leq R_{\langle c, d\rangle},
\end{array}
$$

where

$$
R_{\langle a, b\rangle}=0.5(2-a-b) 0.5(|1-a|+|b|+|1-a-b|)
$$

B. Definition, Operations and Relations over Extended Intuitionistic Fuzzy Index Matrices

Let $\mathscr{I}$ be a fixed set. The definition of twodimensional extended intuitionistic fuzzy IM (2-D EIFIM) $\left[K^{*}, L^{*},\left\{\left\langle\mu_{k_{i}, l_{j}}, v_{k_{i}, l_{j}}\right\rangle\right\}\right]$ with sets $K$ and $L(K, L \subset \mathscr{I})$ is [12]: 


\begin{tabular}{l|ccc} 
& $l_{1},\left\langle\alpha_{1}^{l}, \beta_{1}^{l}\right\rangle$ & $\ldots$ & $l_{n},\left\langle\alpha_{n}^{l}, \beta_{n}^{l}\right\rangle$ \\
\hline$k_{1},\left\langle\alpha_{1}^{k}, \beta_{1}^{k}\right\rangle$ & $\left\langle\mu_{k_{1}, l_{1}}, v_{k_{1}, l_{1}}\right\rangle$ & $\ldots$ & $\left\langle\mu_{k_{1}, l_{n}}, v_{k_{1}, l_{n}}\right\rangle$ \\
$\vdots$ & $\vdots$ & $\ldots$ & $\vdots$ \\
$k_{i},\left\langle\alpha_{i}^{k}, \beta_{i}^{k}\right\rangle$ & $\left\langle\mu_{k_{i}, l_{1}}, v_{k_{i}, l_{1}}\right\rangle$ & $\ldots$ & $\left\langle\mu_{k_{i}, l_{n}}, v_{k_{i}, l_{n}}\right\rangle$ \\
$\vdots$ & $\vdots$ & $\ldots$ & $\vdots$ \\
$k_{m},\left\langle\alpha_{m}^{k}, \beta_{m}^{k}\right\rangle$ & $\left\langle\mu_{k_{m}, l_{1}}, v_{k_{m}, l_{1}}\right\rangle$ & $\ldots$ & $\left\langle\mu_{k_{m}, l_{n}}, v_{k_{m}, l_{n}}\right\rangle$
\end{tabular}

where for every $1 \leq i \leq m, 1 \leq j \leq n: \mu_{k_{i}, l_{j}}, v_{k_{i}, l_{j}}, \mu_{k_{i}, l_{j}}+v_{k_{i}, l_{j}} \in$ $[0,1] ; \alpha_{i}^{k}, \beta_{i}^{k}, \alpha_{i}^{k}+\beta_{i}^{k} \in[0,1] ; \alpha_{j}^{l}, \beta_{j}^{l}, \alpha_{j}^{l}+\beta_{j}^{l} \in[0,1]$ and $K^{*}=$ $\left\{\left\langle k_{i}, \alpha_{i}^{k}, \beta_{i}^{k}\right\rangle \mid k_{i} \in K\right\}=\left\{\left\langle k_{i}, \alpha_{i}^{k}, \beta_{i}^{k}\right\rangle \mid 1 \leq i \leq m\right\}$,

$L^{*}=\left\{\left\langle l_{j}, \alpha_{j}^{l}, \beta_{j}^{l}\right\rangle \mid l_{j} \in L\right\}=\left\{\left\langle l_{j}, \alpha_{j}^{l}, \beta_{j}^{l}\right\rangle \mid 1 \leq j \leq n\right\}$.

In [12] are defined the following operations over two EIFIMs $A=\left[K^{*}, L^{*},\left\{\left\langle\mu_{k_{i}, l_{j}}, v_{k_{i}, l_{j}}\right\rangle\right\}\right]$ and $B=\left[P^{*}, Q^{*},\left\{\left\langle\rho_{p_{r}, q_{s}}, \sigma_{p_{r}, q_{s}}\right\rangle\right\}\right]$ : negation, addition-(o,*), termwise subtraction-(max,min), termwise multiplication(min,max), transposition, reduction, projection and substitution. We recall only index type and aggregation operations, and internal subtraction with IMs.

Index type operations [27]: $\operatorname{Index}_{\left\{\left(\min _{R} / \max _{R}\right)\right\}(\not), k_{i}}(A)$ finds the indices of the minimum/ maximum IFFP of the $k_{i}$-th row of $A$ with no empty value in accordance with the relations (2). Index $_{(\max \mu(v)), k_{i}}(A)$ finds the indices of the IFFP of the $k_{i}$-th row of $A$, for which $\mu(v)_{k_{i}, l_{v_{x}}}$ is maximum.

AGIndex $\left\{\left(\min _{R} / \max _{R}\right)\right\}(\notin F)(\not)(\langle 0,1\rangle)(\mu \neq 0)(A)$ finds the indices of the minimum/ maximum element between the elements of $A$, whose indices respectively $\notin F$ or with no empty value, or not equal to $\langle 0,1\rangle$, or with non-zero degree of membership in accordance with the relations (2).

Let us define the following new index type operations: $\operatorname{Index}_{(\langle m, n\rangle)}(A)=\left\{\left\langle k_{w_{1}}, l_{v_{1}}\right\rangle, \ldots,\left\langle k_{w_{y}}, l_{v_{y}}\right\rangle, \ldots,\left\langle k_{w_{W}}, l_{v_{V}}\right\rangle\right\}$, where $\left\langle k_{w_{y}}, l_{v_{y}}\right\rangle$ (for $1 \leq i \leq m$ ) are the indices of the elements equal to the IFP $\langle m, n\rangle$ of $A$.

$A G I n d e x_{\left\{\left(\min _{R} / \max _{R}\right)\right\}(\notin F) /(\not) /(\neq\langle 0,1\rangle)}^{1}(A)$ determines the index of the minimum/ maximum element between the elements $\left\langle\alpha_{i}^{k}, \beta_{i}^{k}\right\rangle$ of $K^{*}$ (the first dimension of $A$ ), whose indices respectively $\notin F$ or with no empty value, or not equal to $\langle 0,1\rangle$ in accordance with the relations (2).

AGIndex ${ }_{\left\{\left(\min _{R} / \max _{R}\right)\right\}(\notin F) /(\not) /(\neq\langle 0,1\rangle)}(A)$ determines the index of the minimum/ maximum element between the elements $\left\langle\alpha_{j}^{l}, \beta_{j}^{l}\right\rangle$ of $L^{*}$ (the second dimension of $A$ ), whose indices respectively $\notin F$ or with no empty value, or not equal to $\langle 0,1\rangle$ in accordance with the relations (2).

Aggregation operations over EIFIMs

We use the operations $\#_{q},(q \leq i \leq 3)$ in aggregation evaluations [26] over IFPs $x=\langle a, b\rangle$ and $y=\langle c, d\rangle$ :

$x \#_{1} y=\langle\min (a, c), \max (b, d)\rangle$;

$x \#_{2} y=\langle$ average $(a, c)$, average $(b, d)\rangle$;

$x \#_{3} y=\langle\max (a, c), \min (b, d)\rangle$.

Let $k_{0} \notin K^{*}$ be a fixed index. Following [12], [26], another form of the defined aggregation operation in [12] by $K$ is:

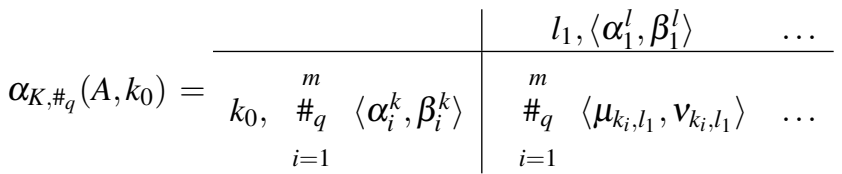

$$
\begin{aligned}
& \begin{array}{ll}
\ldots & l_{n},\left\langle\alpha_{n}^{l}, \beta_{n}^{l}\right\rangle \\
\ldots \quad \#_{q}\left\langle\mu_{k_{i}, l_{n}}, v_{k_{i}, l_{n}}\right\rangle
\end{array} .
\end{aligned}
$$

Aggregate global internal operation: $A G I O_{\oplus_{(\max \min )}}(A)$. Internal subtraction of IMs' components ([24], [25], [27]): $I O_{-(\max , \min )}\left(\left\langle k_{i}, l_{j}, A\right\rangle,\left\langle p_{r}, q_{s}, B\right\rangle\right)=\left[K, L,\left\{\left\langle\gamma_{t_{u}, v_{w}}, \delta_{t_{u}, v_{w}}\right\rangle\right\}\right]$, $\left\langle\gamma_{t_{u}, v_{w}}, \delta_{t_{u}, v_{w}}\right\rangle$

$$
= \begin{cases}\left\langle\mu_{t_{u}, v_{w}}, v_{t_{u}, v_{w}}\right\rangle, & \text { if } t_{u} \neq k_{i} \in K, \\ \left\langle\max \left(0, \mu_{k_{i}, l_{j}}-\rho_{p_{r}, q_{s}}\right),\right. & v_{w} \neq l_{j} \in L ; \\ \left.\min \left(1, v_{k_{i}, l_{j}}+\sigma_{p_{r}, q_{s}}, 1-\mu_{k_{i}, l_{j}}+\rho_{p_{r}, q_{s}}\right)\right\rangle & \text { if } t_{u}=k_{i} \in K, \\ v_{w}=l_{j} \in L\end{cases}
$$

\section{Intuitionistic Fuzzy Graphs (IFGs)}

Let $A$ be an IFS over $E_{1}$ and $B-$ over $E_{2}$. In [11], [22] are defined six versions of the Cartesian products of two IFSs.

The concept of the IFG was introduced in 1994 in [4]. Let us have a fixed set of vertices $\mathscr{V}=\left\{v_{1}, v_{2}, \ldots, v_{n}\right\}$. An (o)-IFG $G($ over $\mathscr{V})$ is the ordered pair $G=\left(V^{*}, A^{*}\right)$, where

$$
\begin{gathered}
V \subset \mathscr{V}, V^{*}=\left\{\left\langle v, \mu_{V}(v), v_{V}(v)\right\rangle \mid v \in V\right\}, \\
A \subset V \times V, A^{*}=\left\{\left\langle\langle x, y\rangle, \mu_{A}(x, y), v_{A}(x, y)\right\rangle \mid\langle x, y\rangle \in V \times V\right\}
\end{gathered}
$$

and functions $\mu_{V}: \mathscr{V} \rightarrow[0,1]$ and $v_{V}: \mathscr{V} \rightarrow[0,1]$ define the degree of membership (existence) and the degree of non-membership (non-existence), respectively, of the element $v \in \mathscr{V}$ to the set $V$; functions $\mu_{A}: E_{1} \times E_{2} \rightarrow[0,1]$ and $v_{A}: E_{1} \times E_{2} \rightarrow[0,1]$ define the degree of membership and the degree of non-membership, respectively, of the element $\langle x, y\rangle \in E_{1} \times E_{2}$ to the set $A \subseteq E_{1} \times E_{2}$; these functions have the forms of the corresponding components of the o-Cartesian product over IFSs from [11], [22] and for all $\langle x, y\rangle \in E_{1} \times E_{2}$,

$$
0 \leq \mu_{V}(x)+v_{V}(x) \leq 1,0 \leq \mu_{A}(x, y)+v_{A}(x, y) \leq 1 .
$$

The all parameters of the IFG $G$ are IFPs. The expert approach described in detail in [11] may be used to determine the distances between any two vertices and the existence of the vertices of the graph in the form of IFPs.

Now, for the graph $G=(V, A)$ was constructed the Extended Intuitionistic Fuzzy Graph (EIFG) $G^{*}=\left(V^{*}, A^{*}\right)$ [12]. It has the following IM-representation as adjacency EIFIM $C$ :

$$
\begin{array}{l|ccc}
\multicolumn{4}{c}{\left[V^{*}, V^{*},\left\{\left\langle\mu\left(v_{i}, v_{j}\right), v\left(v_{i}, v_{j}\right)\right\rangle\right\}\right]} \\
& v_{1},\left\langle\alpha\left(v_{1}\right), \beta\left(v_{1}\right)\right\rangle & \ldots & v_{n},\left\langle\alpha\left(v_{n}\right), \beta\left(v_{n}\right)\right\rangle \\
\hline v_{1},\left\langle\alpha\left(v_{1}\right), \beta\left(v_{1}\right)\right\rangle & \left\langle\mu_{v_{1}, v_{1}}, v_{v_{1}, v_{1}}\right\rangle & \ldots & \left\langle\mu_{v_{1}, v_{n}}, v_{v_{1}, v_{n}}\right\rangle \\
\vdots & \vdots & \ldots & \vdots \\
v_{i},\left\langle\alpha\left(v_{i}\right), \beta\left(v_{i}\right)\right\rangle & \left\langle\mu_{v_{i}, v_{1}}, v_{v_{i}, v_{1}}\right\rangle & \ldots & \left\langle\mu_{v_{i}, v_{n}}, v_{v_{i}, v_{n}}\right\rangle \\
\vdots & \vdots & \ldots & \vdots \\
v_{n},\left\langle\alpha\left(v_{n}\right), \beta\left(v_{n}\right)\right\rangle & \left\langle\mu_{v_{n}, v_{1}}, v_{v_{n}, v_{1}}\right\rangle & \ldots & \left\langle\mu_{v_{n}, v_{n}}, v_{v_{n}, v_{n}}\right\rangle
\end{array},
$$$$
\text { where for every } 1 \leq i \leq n, 1 \leq j \leq n \text { : } C_{v_{i}, v_{j}}=\left\langle\mu_{v_{i}, v_{j}}, v_{v_{i}, v_{j}}\right\rangle
$$
and $\left\langle\alpha\left(v_{i}\right), \beta\left(v_{i}\right)\right\rangle$ are IFPs. 
Proposition 1 In an IFG, if every vertex has exactly two adjacent vertices, then there exists a Hamiltonian cycle.

The proof of the proposition is analogous to that of [1].

\section{Algorithms FOR HAMiltonian CYCLE IN AN IFG}

Let us be given EIFG $G^{*}=\left(V^{*}, A^{*}\right)$ with the IMrepresentation as EIFIM $C$ with a structure (3). The purpose is to find the Hamiltonian cycle in $G^{*}$. Let us extend the algorithms for Hamiltonian cycle in a fuzzy graph from [1] to intuitionistic fuzzy ones, based on IFSs and IMs concepts. Algorithm 1: Minimum edge degree algorithm to find intuitionistic fuzzy Hamiltonian cycle

\begin{tabular}{|c|c|c|c|}
\hline \multicolumn{4}{|c|}{ Let us define $X\left[V^{*}, V^{*},\left\{\left\langle\rho\left(v_{i}, v_{j}\right), \sigma\left(v_{i}, v_{j}\right)\right\rangle\right\}\right]$} \\
\hline & $v_{1},\left\langle\alpha\left(v_{1}\right), \beta\left(v_{1}\right)\right\rangle$ & $\ldots$ & $v_{n},\left\langle\alpha\left(v_{n}\right), \beta\left(v_{n}\right)\right\rangle$ \\
\hline$v_{1},\left\langle\alpha\left(v_{1}\right), \beta\left(v_{1}\right)\right\rangle$ & $\left\langle\rho_{v_{1}, v_{1}}, \sigma_{v_{1}, v_{1}}\right\rangle$ & $\ldots$ & $\left\langle\rho_{v_{1}, v_{n}}, \sigma_{v_{1}, v_{n}}\right\rangle$ \\
\hline$\vdots$ & $\vdots$ & & $\vdots$ \\
\hline$v_{n},\left\langle\alpha\left(v_{n}\right), \beta\left(v_{n}\right)\right\rangle$ & $\left\langle\rho_{v_{n}, v_{1}}, \sigma_{v_{n}, v_{1}}\right\rangle$ & $\cdots$ & $\left\langle\rho_{v_{n}, v_{n}}, \sigma_{v_{n}, v_{n}}\right\rangle$ \\
\hline
\end{tabular}

where for $i$ and $j: x_{v_{i}, v_{j}}$ and $\left\langle\alpha\left(v_{i}\right), \beta\left(v_{i}\right)\right\rangle$ are IFPs.

Let us we create the following auxiliary IMs:

1) $S=\left[V^{*}, V^{*},\left\{s_{k_{i}, l_{j}}\right\}\right]$, such that $S=C$ i.e.

$\left(s_{k_{i}, l_{j}}=c_{k_{i}, l_{j}} \forall k_{i} \in V^{*}, \forall l_{j} \in V^{*}\right)$;

2)

$$
R C\left[V^{*}, e_{0}\right]=\begin{array}{c|c} 
& e_{0} \\
\hline k_{1} & r c_{k_{1}, e_{0}} \\
\vdots & \vdots \\
k_{n} & r c_{k_{n}, e_{0}}
\end{array},
$$

where for $1 \leq i \leq n: \quad r c_{k_{i}, e_{0}}=\{0,1\}$ depending on whether the $k_{i}$-th vertex of the matrix $S$ is crossed out (introduced in the Hamiltonian path) or not. When the algorithm starts, $r c_{k_{i}, e_{0}}=0, x_{v_{i}, v_{j}}=\langle 0,1\rangle\left(\forall k_{i} \in V *, \forall l_{j} \in V *\right)$.

We will propose the algorithm for determining a Hamiltonian path in $G^{*}$, interpreted with the tools of IMs and IFPs extending the fuzzy algorithm from [1]:

Step 1. Construct the EIFIM $C$ for the given IFG $G^{*}$ and create EIFIM $S$ such that $S:=C$; Check the condition of the proposition 1. for the existence of a Hamiltonian cycle in $G^{*}$ : for $i=1$ to $n$ then $\left\{\operatorname{AGIndex}_{\left\{\left(\min _{R} / \max _{R}\right)\right\}(\mu \neq 0)}(S)=\right.$ $\left.\left\{\left\langle k_{w_{i}}, l_{v_{1}}\right\rangle, \ldots,\left\langle k_{w_{i}}, l_{v_{y}}\right\rangle, \ldots,\left\langle k_{w_{i}}, l_{v_{V}}\right\rangle\right\}\right\}$.

If $V<2$ then the Hamiltonian path does not exist and the algorithm Stop\}

else Go to Step 2\}.

Step 2. Search for a minimum IFP in the $S$ with non-zero membership degree in accordance with the relations (2). If there are several such elements, then we choose any one.

AGIndex $_{\left\{\left(\min _{R}\right)\right\}(\mu \neq 0)}(S)=\left\langle k_{z}, l_{z}\right\rangle$.

If $x_{k_{z}, l_{z}}=\langle 0,1\rangle$, then $\left\{r c\left[k_{z}, e_{0}\right]=1 ; S_{\left(k_{i}, \perp\right)}\right\}$

$x_{k_{z}, l_{z}}=\langle 1,0\rangle\left\{r c\left[k_{i}, e_{0}\right]=1 ; S_{\left(k_{i}, \perp\right)}\right\} ;$ Go to Step 3 .

Step 3. If the minimum IFP $s_{k_{z}, l_{z}}$ in the IM $S$ does not allow for a fuzzy Hamiltonian path, then the next higher minimum IFP is selected.

AGIndex $_{\left\{\left(\min _{R}\right)\right\}(\mu \neq 0)}(S)=\left\langle k_{x}, l_{x}\right\rangle$; Go to Step 4 .

Step 4. Identify the $k_{x}$-th row and $l_{x}$-th column in the $S$ where the minimum IFP appears. We add to Hamiltonian the vertex $l_{x}$ i.e. from $k_{x}$ it reaches $l_{x} \cdot r c_{k_{x}, e_{0}}=r c_{l_{x}, e_{0}}=1$, $x_{k_{x}, l_{x}}=\langle 1,0\rangle$ and $S$ is reduced by $S_{\left(\perp, l_{x}\right)}$; Go to Step 5 .

Step 5. Search for a minimum IFP of the $l_{x}$-th row, such that: it forms a fuzzy Hamiltonian path; if the minimum value occurs more than once, then an IFP is selected for a Hamiltonian path

The operation is: $A G \operatorname{Index}_{\left\{\left(\min _{R}\right)\right\}(\mu \neq 0)}\left(p r_{l_{x}, V^{*}} S\right)=\left\langle l_{x}, l_{u}\right\rangle$;

Then $r c_{l_{x}, e_{0}}=r c_{l_{u}, e_{0}}=1, \quad x_{k_{x}, l_{u}}=\langle 1,0\rangle$ and the IM $S$ is reduced by $S_{\left(l_{x}, l_{u}\right)}$.

Step 6. Repeat Step 3 through Step 4 row-wise until $\mid$ Index $_{(1)}(R C) \mid=n$. With $|A|$ let us we denote the number of elements of the $A$, where $A$ is an IM.

If $\left|\operatorname{Index}_{(1)}(R C)\right|=n$ then an intuitionistic fuzzy Hamiltonian path with all $n$ vertices of $G^{*}$ is found and go to Step 7, else there is no intuitionistic fuzzy Hamiltonian path and repeat Step 2 or Step 3 as required.

Step 7. If intuitionistic fuzzy Hamiltonian path exists, then only one row $k_{l}$ will be left out in the IM $S$. Select an IFP with non-zero degree of membership from that row to form a fuzzy Hamiltonian cycle, if exists. If we can select the IFP $s_{k_{l}, k_{z}}$ with non-zero membership degree then $\{$ the Hamiltonian cycle exists, $x_{k_{l}, k_{z}}=\langle 1,0\rangle$ and go to Step 8\}

else the algoritm Stop.

Step 8. If $\left|\operatorname{Index}_{(\langle m, n\rangle)}(X)\right|=n$, then the optimal Hamiltonian path is obtained with minimum intuitionistic fuzzy length according to (2). The IF length of the path is:

$A G I O_{1_{\oplus_{(\max , \min )}}}\left(C \otimes_{(\min , \max )} X\right)$ or $A G I O_{2_{\oplus}\left(\mathrm{V}_{2}\right)}\left(C \otimes_{\left(\wedge_{2}\right)} X\right)$, where $\vee_{2}$ and $\wedge_{2}$ are the operations from (1).

For an IFG with $n$ vertices the algorithm visits all the permutations of the vertices, so the complexity is $O(n !)$.

Algorithm 2 Minimum vertex degree algorithm to find an intuitionistic fuzzy Hamiltonian cycle

Step 1. We select a vertex $v_{\text {min }, i}$, whose $\left\langle\alpha\left(v_{\text {min }, i}\right), \beta\left(v_{\text {min, }, i}\right)\right\rangle$ is the minimum IFPs $\left\langle\alpha\left(v_{i}\right), \beta\left(v_{i}\right)\right\rangle(1 \leq i \leq n)$. (If there is more than one vertex with same IFP, then choose any one).

Step 2. Select a vertex with IFP whose is next higher to the minimum IFP $\left\langle\alpha\left(v_{\min , i}\right), \boldsymbol{\beta}\left(v_{\min , i}\right)\right\rangle$.

Step 3. Identify the adjacent vertices of the vertex with minimum IFP selected.

Step 4. We select the unvisited adjacent vertex of the minimum IFP $\left\langle\alpha\left(v_{i}\right), \beta\left(v_{i}\right)\right\rangle(1 \leq i \leq n)$. (If more than one adjacent vertex has the same minimum IFP, then choose any one vertex).

Step 5. Step 3 - Step 4 are repeated until an IF Hamiltonian cycle is found else go to Step 1. or Step 2. as required.

This algorithm 2 can be presented with similar IMs operations to that of algorithm 1, but the operation AGIndex $\left\{\left(\min _{R} / \max _{R}\right)\right\}(\mu \neq 0)(S)$ will be used instead AGIndex $\left\{\left(\min _{R} / \max _{R}\right)\right\}(\mu \neq 0)(S)$. In the algorithm 2, step 3 and step 4 need to be repeated starting with each vertex of IFG $G^{*}$ to find all possible IF Hamiltonian cycles. We can identify the minimum length of IF Hamiltonian cycle(s).

\section{AN EXAMPLE FOR HAMILTONIAN CYCLE IN IFG}

The part of the airline network of the Wizz air is modeled by an IFG $G^{*}=\left(V^{*}, A^{*}\right)$. The vertices of IFG are \{Sofia airport (S), Dortmund airport (D), Viena airport (V), Brussels South 
Charleroi Airport (Br), Barcelona airport (Ba)\}. The IFG has the following IM-representation as EIFIM $C\left[V^{*}, V^{*}\right]$ :

\begin{tabular}{c|ccc} 
& $S,\langle 0.6 ; 0.20\rangle$ & $D,\langle 0.75 ; 0.20\rangle$ & $\ldots$ \\
\hline$S,\langle 0.6 ; 0.20\rangle$ & $\langle 0 ; 1\rangle$ & $\langle 0.80 ; 0.18\rangle$ & $\ldots$ \\
$D,\langle 0.75 ; 0.20\rangle$ & $\langle 0.80 ; 0.18\rangle$ & $\langle 0 ; 1\rangle$ & $\ldots$ \\
$V,\langle 0.70 ; 0.1\rangle$ & $\langle 0,40 ; 0.50\rangle$ & $\langle 0,35 ; 0.60\rangle$ & $\ldots$ \\
$B r,\langle 0.80 ; 0.10\rangle$ & $\langle 0,80 ; 0.10\rangle$ & $\langle 0,10 ; 0.90\rangle$ & $\ldots$ \\
$B a,\langle 0.83 ; 0.15\rangle$ & $\langle 0.90 ; 0.05\rangle$ & $\langle 0.50 ; 0.48\rangle$ & $\ldots$ \\
$\ldots$ & $V,\langle 0.70 ; 0.1\rangle$ & $B r,\langle 0.80 ; 0.10\rangle$ & $B a,\langle 0.83 ; 0.15\rangle$ \\
\hline$\ldots$ & $\langle 0.40 ; 0.50\rangle$ & $\langle 0.80 ; 0.10\rangle$ & $\langle 0.90 ; 0.05\rangle$ \\
$\ldots$ & $\langle 0.35 ; 0.60\rangle$ & $\langle 0.10 ; 0.90\rangle$ & $\langle 0.50 ; 0.48\rangle$ \\
$\ldots$ & $\langle 0 ; 1\rangle$ & $\langle 0.38 ; 0.60\rangle$ & $\langle 0.75 ; 0.20\rangle$ \\
$\ldots$ & $\langle 0.38 ; 0.60\rangle$ & $\langle 0 ; 1\rangle$ & $\langle 0.45 ; 0.50\rangle$ \\
$\ldots$ & $\langle 0.75 ; 0.20\rangle$ & $\langle 0.45 ; 0.50\rangle$ & $\langle 0 ; 1\rangle$
\end{tabular}.

The IFPs, presented the vertices and the edges of $G^{*}$ are calculated using the expert approach [11]. Each of the experts is asked to evaluate the degree of membership of IFP, corresponding to every vertex using the ratio of the air distance to all destinations from it to the total air distance of all the air roads. The minimum degree of membership proposed by the experts for the respective vertex is the degree of membership for this vertex. The degree of non-membership of the vertex is calculted as 1-maximum degree of membership, proposed by the experts. The approach to calculating the IFP for the length of each edge is similar. Each expert estimates the degree of membership of IFP, corresponding to every edge using the ratio of the air distance between two cities to the total air distance of all the air roads in the map of Wizz air. Let us find a Hamiltonian cycle in the $G^{*}$ using the algorithm 1:

Step 1. Let us create EIFIM $C$ for the given IFG $G^{*}$. Then we create IM $S$ such that $S:=C$. The condition of the proposition 1 for the existence of a Hamiltonian cycle in $G^{*}$ is met.

Step 2. The minimum IFP in the $S$ with non-zero membersheep degree is $s_{D, V}=\langle 0.35 ; 0.60\rangle$.

Step 3. The minimum IFP in the $S$ represents that from Dortmund airport it reaches Vienna airport.

Step 4. In the row "V" the minimum IFP is equal to $\langle 0.38 ; 0.60\rangle=s_{V, B r}$. Therefore from Vienna airport it goes to Brussels South Charleroi Airport.

Step 5. Repeat Steps 3-4, the path obtained is: D-V-Br-Ba-S. Step 6. From the row "S", select the element $s_{S, D}=$ $\langle 0.80 ; 0.18\rangle$ with non-zero membership degree to get an IF Hamiltonian cycle "D-V-Br-Ba-S-D." The IF length of the path is: $A G I O_{1_{\oplus(\max , \min )}}\left(C \otimes_{(\min , \max )} X\right)=\langle 0.9 ; 0.1\rangle$.

\section{CONCLUSION}

In this paper it was developed new methods to obtain the IF Hamiltonian cycle in an IFG, using the IFSs and IMs concepts. The main contribution of our approach lies in its ability to find a Hamiltonian cycle not only in a clear but also in an uncertain environment. The proposed algorithms can be generalized to multidimensional intuitionistic fuzzy data [13]. The efficiency of the first algorithm was demonstrated by a real data example from the selected network map of Wizz air. In the paper also was defined three index type operations over EIFIMs. In the future, we will extend this algorithm for an application to the interval-valued intuitionistic fuzzy graphs [14].

\section{REFERENCES}

[1] A. Gani, S. Latha, "A new algorithm to find fuzzy Hamilton cycle in a fuzzy network using adjacency matrix and minimum vertex degree," SpringerPlus, International Journal of Pure and Applied Mathematics, vol. 5, 2016, 1854.

[2] A. Kauffman, Introduction a la Theorie des Sous-emsembles Flous, Paris: Masson et Cie Editeurs, 1973.

[3] A. Rosenfeld, "Fuzzy graphs," in: L.A. Zadeh, K.S. Fu, M. Shimura (Eds.), Fuzzy Sets and Their Applications, Academic Press, New York, 1975, pp. 77-95.

[4] A. Shannon, K. Atanassov, "A first step to a theory of the intuitionistic fuzzy graph," in D. lakov, ed., Proc. of the First workshop on Fuzzy based expert systems, Sofia, 1994, pp. 59-61.

[5] E. Szmidt, J. Kacprzyk, "Amount of information and its reliability in the ranking of Atanassov's intuitionistic fuzzy alternatives," in: RakusAndersson and etc. (eds.), Recent Advances in Decision Making, SCI, Springer, Heidelberg, vol. 222, 2009, pp. 7-19.

[6] GA. Dirac, "Some theorems on abstract graphs," Proc Lond Math Soc, vol. 3 (1), 1952, pp. 69-81.

[7] G. Fan, "New sufficient conditions for cycles in graphs," J Comb Theory Ser B, vol. 37, 1984, pp. 221-227.

[8] JN. Mordeson, PS. Nai, "Cycles and co-cycles of fuzzy graphs," Inf Sci, vol. 90, 1996, pp. 39-49.

[9] K. Atanassov, "Intuitionistic Fuzzy Sets," VII ITKR Session, Sofia, 2023 June 1983.

[10] K. Atanassov, "Generalized index matrices," Comptes rendus de l'Academie Bulgare des Sciences, vol. 40(11), 1987, pp. 15-18.

[11] K. Atanassov, On Intuitionistic Fuzzy Sets Theory, STUDFUZZ. Springer, Heidelberg, vol. 283; 2012.

[12] K. Atanassov, Index Matrices: Towards an Augmented Matrix Calculus. Studies in Computational Intelligence, Springer, Cham, vol. 573; 2014.

[13] K. Atanassov, "n-Dimensional extended IMs Part 1," Advanced Studies in Contemporary Mathematics, vol. 28 (2), 2018, pp. 245-259.

[14] K. Atanassov, Interval-valued intuitionistic fuzzy sets, Studies in Fuzziness and Soft Computing, vol. 388; 2020.

[15] K. Atanassov, E. Szmidt, J. Kacprzyk, "On intuitionistic fuzzy pairs," Notes on Intuitionistic Fuzzy Sets, vol. 19 (3), 2013, pp. 1-13.

[16] K. Kathirvel, K. Balamurugan, "Method for solving fuzzy transportation problem using trapezoidal fuzzy numbers," International Journal of Engineering Research and Applications, vol. 2 (5), 2012, pp. 2154-2158.

[17] K. Zhao, Lai Hong-Jian, Shao Yehang, "New sufficient condition for Hamiltonian graphs," Appl Math Lett, vol. 20, 2007, 116-122.

[18] L. Zadeh, Fuzzy Sets, Information and Control, vol. 8 (3), 338-353; 1965.

[19] O. Ore, "Note on Hamiltonian circuits." Am Math Mon, vol. 67, 1960, pp. 55.

[20] RM. Karp, "Reducibility among combinatorial problems," in: Miller RE, Thatcher JW (eds), Complexity of computations, Plemem Press, New York, 1972, pp. 85-103.

[21] S. Skiena, Hamiltonian cycles. Implementing discrete mathematics: combinatorics and graph theory with mathematica reading, Addison Wesley, New York; 1990, pp 196-198.

[22] V. Andonov, "On some properties of one Cartesian product over Intuitionistic fuzzy sets." Notes on Intuitionistic Fuzzy Sets, vol. 14 (1), 2008,12-19.

[23] V. Chvatal, “On Hamilton's ideals,” J Combin Theory Ser B, vol. 12, 1972, pp. 63-168.

[24] V. Traneva, "Internal operations over 3-dimensional extended index matrices," Proceedings of the Jangjeon Mathematical Society, vol. 18 (4), 2015, pp. 547-569.

[25] V. Traneva, S. Tranev, V. Atanassova, "An Intuitionistic Fuzzy Approach to the Hungarian Algorithm," in: G. Nikolov et al. (Eds.): NMA 2018, LNCS 11189, Springer Nature Switzerland, AG, 2019, pp. 1-9.

[26] V. Traneva, S. Tranev, M. Stoenchev, K. Atanassov, " Scaled aggregation operations over two- and three-dimensional index matrices," Soft computing, vol. 22, 2019, pp. 5115-5120.

[27] V. Traneva, S. Tranev, Index Matrices as a Tool for Managerial Decision Making, Publ. House of the Union of Scientists, Bulgaria; 2017

[28] http://wizz.air-bg.com, last accessed 29 june 2020. 